\title{
Performances des exploitations laitières familiales au Maroc : arguments pour I'amélioration du prix du lait à la ferme et de l'appui technique
}

\author{
M.T. Sraïri ${ }^{1 *}$ S. Chergui $^{1}$ H. Igueld ${ }^{1}$ Y. Sannito ${ }^{1}$
}

Mots-clés

Bovin - Vache laitière - Rendement laitier - Coût de production - Ration Durabilité - Rentabilité - Maroc.

\begin{abstract}
Résumé
L'élevage bovin laitier au Maroc a connu ces dernières années une évolution mouvementée. Cette activité contribue pourtant fortement à l'approvisionnement des laiteries et des marchés urbains, ainsi qu'au maintien des conditions de vie en zone rurale. La présente étude a eu pour objectif de caractériser les performances techniques et économiques de l'élevage bovin au regard des contraintes qu'il doit surmonter. Dans une première partie, le prix de revient (coût de production) du lait a été étudié sur la base d'une enquête conduite dans 86 étables réparties dans les principaux bassins d'élevage du Maroc. Deux méthodes de calcul ont permis de porter un jugement sur la rentabilité de cette activité. Dans une seconde partie, les manques à gagner issus de rendements laitiers limités ont été caractérisés et mis en relation avec les rations ingérées par les vaches en lactation dans six étables, au cours de deux saisons successives (le printemps et l'été) dans une région favorable du pays (le périmètre irrigué du Gharb). Ce travail a souligné la faible rentabilité des fermes bovines et les marges $d^{\prime}$ 'amélioration en matière de rationnement, surtout pour les vaches à haut potentiel. Le conseil aux éleveurs permettant d'améliorer les pratiques d'alimentation des animaux et la revalorisation du prix de vente du lait à la ferme constituent deux conditions importantes du maintien durable de l'élevage bovin laitier au Maroc.
\end{abstract}

\section{INTRODUCTION}

L'élevage bovin constitue une activité importante de la production agricole au Maroc, représentant près de 7,5 p. 100 du produit intérieur brut agricole (1). Il est fortement associé aux espaces agraires, à la différence des petits ruminants, plus localisés dans des zones de parcours non cultivés. Les multiples rôles assumés par les bovins au Maroc (création d'emplois et de revenus, approvisionnement des marchés en lait et viande, maintien de la fertilité des sols par épandage de fumier, épargne des familles paysannes, etc.) en font un élément clé du développement agricole du pays.

1. Département des productions et biotechnologies animales, Institut agronomique et vétérinaire Hassan II, BP 6202, Madinate Al Irfane, Rabat, Maroc.

* Auteur pour la correspondance

Tél. : + 212537771758

E-mail : mt.srairi@iav.ac.ma
Ainsi, depuis l'Indépendance en 1956, et pour faire face à l'accroissement rapide et continu des besoins nutritionnels induits par l'expansion démographique et les évolutions sociales (par exemple urbanisation, affirmation des individualismes, repas à l'extérieur du domicile), des plans ont visé la promotion de l'élevage bovin $(14,15)$. Ceux-ci ont comporté une panoplie de mesures comme l'importation de races laitières, la production de fourrages en zones irriguées, la vulgarisation de l'insémination artificielle ou la mécanisation de la traite. Ces politiques ont aussi toutes appuyé un approvisionnement des marchés en produits bovins (lait et viande) prioritairement issus d'élevages locaux, fournissant des opportunités d'emplois dans le monde rural. Ainsi, à la différence de pays limitrophes, le Maroc a fortement limité ses importations de lait et de viande par une protection douanière conséquente (24). En outre, l'installation d'une infrastructure de collecte du lait disséminée à travers le territoire offre aux éleveurs une garantie de revenus quotidiens. 
La structure de l'élevage bovin au Maroc repose sur un nombre élevé d'exploitations (près de 450 000), dont une proportion importante caractérisée par une taille restreinte : 75 p. 100 des fermes détiennent moins de cinq vaches sur une surface inférieure à cinq hectares. L'offre atomisée induite par cette structure implique des difficultés évidentes d'accompagnement des éleveurs. De plus, le désengagement des services étatiques des tâches traditionnelles d'appui technique, suite à l'application des mesures de l'ajustement structurel dans les années 1980 (12), a fragilisé une partie des exploitations détenant des bovins puisque la quasiintégralité des services, comme l'insémination artificielle ou la prophylaxie sanitaire, sont devenus payants, cédés à des opérateurs privés (25).

Une autre caractéristique de l'élevage bovin au Maroc est sa vulnérabilité aux aléas climatiques (niveaux et répartition de la pluviométrie annuelle), qui affecte directement les rendements de biomasse fourragère et la rentabilité de cette activité (26). Les productions bovines reflètent largement les fluctuations interannuelles des précipitations, et ceci constitue un handicap majeur pour l'essor durable du secteur. Au regard des changements climatiques que risque de connaître le pays, et qui s'accompagneraient d'aléas plus prononcés, la vulnérabilité des populations rurales pratiquant une agriculture vivrière va s'accroître, induisant davantage de précarité pour près de 50 p. 100 des habitants (22).

Aussi, a-t-il été relevé une tendance nette à l'amplification des usages de ressources alimentaires achetées dans les élevages en cas de conjoncture climatique défavorable (29). La baisse d'autonomie fourragère qui en résulte se traduit par des répercussions négatives aussi bien sur l'économie de la production (augmentation des coûts monétaires) que sur l'état de santé général des vaches (21). Ces retombées économiques négatives se sont considérablement amplifiées à partir de 2008 avec l'accroissement de la volatilité des cours des matières premières alimentaires et des autres intrants (par exemple génisses, machines, vaccins).

Par ailleurs, les changements sociaux dans le monde rural et l'ensemble des tensions politiques actuelles qui agitent certains pays de la région du Maghreb ont induit l'émergence de revendications en faveur de l'amélioration des niveaux de rémunération du travail agricole. Encore peu de travaux de recherche renseignent ces tendances dans les exploitations familiales. Mais il n'est pas exclu que les marges limitées générées par le troupeau bovin, associées aux lourdes astreintes de travail qu'il nécessite, ne conduisent des membres des familles paysannes à vouloir changer d'activités et à opter pour des occupations plus rémunératrices, notamment en accentuant l'exode rural.

Face à ce contexte mouvementé, le présent article visait à renseigner les caractéristiques de durabilité des élevages bovins laitiers au Maroc. Dans un premier temps, le prix de revient du lait dans un échantillon d'élevages représentatifs de la diversité des situations rencontrées dans le pays a été analysé. En utilisant deux méthodes de calcul distinctes, les effets des résultats obtenus sur la durabilité économique et sociale de l'élevage bovin ont été évalués. La deuxième partie de l'article visait à caractériser les manques à gagner des étables laitières à travers une étude de cas concrets des apports alimentaires des vaches par rapport à leurs besoins optimaux au cours de deux saisons successives. Ces deux volets de l'article ont permis de discuter des défis à relever pour pérenniser les conditions d'un développement durable de l'élevage bovin laitier au Maroc. Il s'agit notamment d'évaluer la capacité de cette activité à continuer à participer à l'approvisionnement des marchés locaux, tout en assurant des revenus réguliers et une rentabilité convenable aux familles qui s'y sont engagées.

\section{MATERIEL ET METHODES}

L'étude du prix de revient (coût de production) du lait de vache a été réalisée au cours de deux printemps successifs, en 2012 et 2013, de mars à juin. Au total, 86 exploitations distribuées dans sept régions du pays ont fait l'objet de ce travail (figure 1). Le tableau I montre la diversité des zones agroécologiques. Les caractéristiques structurelles de ces exploitations (surface fourragère, taille du cheptel et charge animale) sont reportées dans le tableau II. L'échantillon reflète la réalité de l'élevage bovin laitier au Maroc, avec une majorité d'exploitations de moins de cinq hectares. Les exploitations étudiées ne sont pas spécialisées dans l'élevage bovin mais ce dernier y occupe un rôle prépondérant, au vu de la part de la surface agricole utile destinée aux fourrages. Les familles élèvent des vaches, surtout de races laitières spécialisées importées (Holstein et Montbéliarde) mais aussi des vaches de type croisé (races locales x races importées).

Outre l'élevage, toutes les exploitations pratiquent la céréaliculture et aussi, pour certaines, des cultures de rente comme le maraîchage

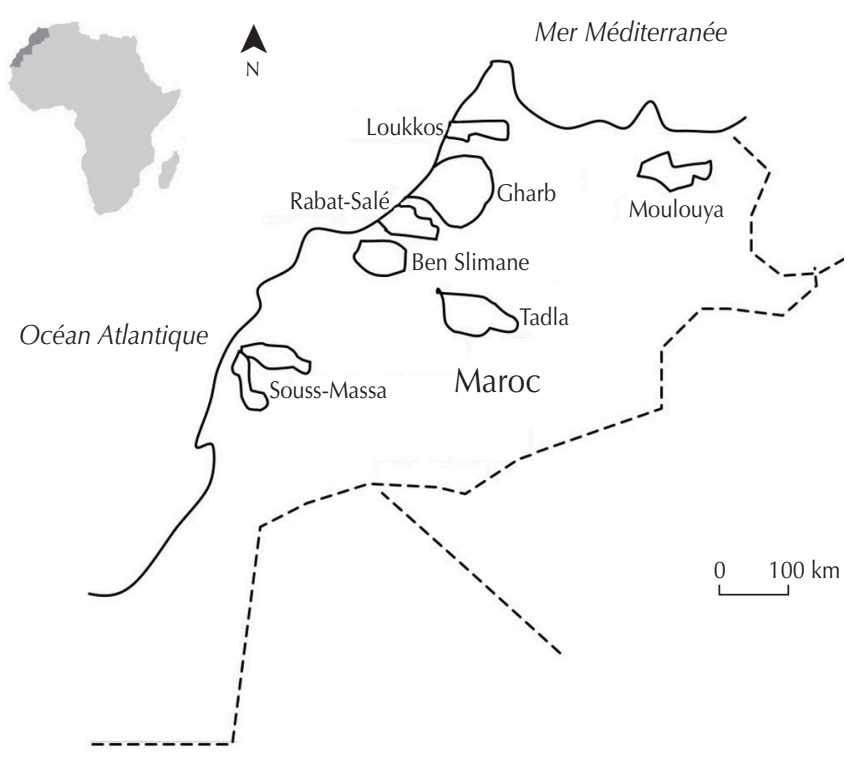

Figure 1 : localisation des régions d'élevage dont le prix de revient du lait a été déterminé.

Tableau I

Répartition des exploitations enquêtées selon la zone agroécologique

\begin{tabular}{|llcc|}
$\begin{array}{l}\text { Zone } \\
\text { agroécologique }\end{array}$ & Région & $\begin{array}{c}\text { Nb. total } \\
\text { d'exploitations } \\
\text { bovines en 2012 }\end{array}$ & $\begin{array}{c}\text { Nb. d'élevages } \\
\text { de l'étude } \\
(\mathbf{n = 8 6})\end{array}$ \\
\hline Suburbaine & $\begin{array}{l}\text { Ben Slimane } \\
\text { Rabat-Salé }\end{array}$ & 3250 & 10 \\
Périmètre de & 21500 & 13 \\
grande hydraulique & Gharb & 26300 & 10 \\
& Loukkos & 14200 & 15 \\
& $\begin{array}{l}\text { Moulouya } \\
\text { Souss-Massa }\end{array}$ & 6300 & 14 \\
& $\begin{array}{l}\text { Tadla } \\
\text { Ta } 500\end{array}$ & 14 \\
& 16900 & 10
\end{tabular}

Adapté par les auteurs à partir de statistiques des directions régionales de l'Agriculture au Maroc 


\section{Tableau II}

Caractéristiques structurelles moyennes des élevages étudiés dans les différentes régions

$\begin{array}{lcccc}\text { Région } & \begin{array}{c}\text { Surface } \\ \text { fourragère } \\ \text { (ha) }\end{array} & \begin{array}{c}\text { Nb. moyen } \\ \text { de vaches/ } \\ \text { exploitation }\end{array} & \begin{array}{c}\text { Charge } \\ \text { animale } \\ \left.\text { (UGB }{ }^{1} / \mathbf{h a}\right)\end{array} & \begin{array}{c}\text { Concentrés } \\ \text { / L de lait } \\ \text { (kg) }\end{array} \\ \text { Ben Slimane } & 3,5 & 6,6 & 2,5 & 0,83 \\ \text { Gharb } & 5,5 & 9,4 & 2,2 & 0,45 \\ \text { Loukkos } & 3,1 & 12,0 & 4,9 & 0,36 \\ \text { Moulouya } & 1,1 & 7,1 & 9,8 & 0,28 \\ \text { Rabat-Salé } & 4,2 & 7,8 & 2,7 & 0,56 \\ \text { Souss-Massa } & 1,9 & 7,7 & 7,2 & 0,75 \\ \text { Tadla } & 1,2 & 6,1 & 8,0 & 0,52\end{array}$

${ }^{1}$ Unité de gros bétail

ou la betterave sucrière. Dans la majorité des cas, les vaches sont en mode de faire valoir direct. Les acquisitions de vaches importées sont généralement financées auprès des industriels laitiers, moyennant un versement initial correspondant à près de 30 p. 100 de la valeur des animaux, tandis que le reste est payé par prélèvement sur le lait livré. En outre, les grandes unités (plus de 30 vaches) ont été exclues de ce travail, du fait des difficultés rencontrées pour accéder aux données relatives à leur fonctionnement. Cette limite de notre étude est discutée dans la conclusion.

Le prix de revient du lait a été calculé à partir des résultats d'une enquête quotidienne conduite dans les exploitations visitées. A cette occasion, l'observation de la structure du cheptel (vaches en lactation et taries, veaux et génisses) et des infrastructures (bâtiments, matériel, entre autres) a permis de renseigner le niveau des immobilisations (capital) de ces exploitations. En outre, les prix de revient des fourrages consommés le jour de l'enquête ont été déterminés par la reconstitution de leur train technique (notamment labour, semences, fertilisation, irrigation) et des frais occasionnés par ces opérations culturales. Les sommes correspondantes ont été divisées par le nombre de jours d'exploitation de ces fourrages. Par ailleurs, les usages et les prix des intrants achetés (aliments concentrés, produits vétérinaires, litière, etc.) et utilisés le jour de l'enquête ont été déterminés. Le volume de lait produit le jour de la visite a été noté. L'inventaire des ventes annuelles de bovins a été dressé car ces ventes constituent un coproduit de la production laitière (20) qui doit être intégré dans le calcul du prix de revient du lait comme l'indique la formule suivante :

prix de revient du lait $=($ total des charges d'élevage bovin - produit des ventes de bovins) / production totale de lait

Le calcul des charges et des produits a été rapporté au volume de lait produit par exploitation pour calculer un prix moyen de revient du litre de lait. Deux méthodes de calcul ont été distinguées. La première intègre, outre les dépenses courantes (aliments, main d'œuvre extra-familiale salariée, frais vétérinaires et d'insémination, électricité, et énergie fossile), les amortissements des investissements et la rémunération de la main-d'œuvre familiale. La seconde ne considère que les charges courantes. Il s'agit donc du prix monétaire réellement payé par les producteurs.

Dans le cas de la première méthode, les amortissements spécifiques aux investissements relatifs à l'élevage (achat d'animaux, bâtiments et matériel d'élevage) ont été déterminés en prenant comme hypothèse une dévaluation linéaire de leur valeur, avec une durée de vie de 20 ans pour les bâtiments, 10 ans pour le matériel lourd (tracteur, charrue, ensileuse, etc.), et 5 ans pour le matériel léger (chariot de traite, motopompe, etc.) et les vaches (avec une valeur vénale variable selon le type génétique : 2200 et $1600 €$ respectivement pour une vache de race importée ou de type croisé). Un coût d'opportunité équivalent au salaire minimal agricole garanti (Smag ; 5,6€ par jour) en vigueur au Maroc a été attribué à la main-d'œuvre familiale dans la première méthode.

Le deuxième volet de cet article, relatif à la caractérisation des manques à gagner des élevages bovins laitiers et leurs relations à l'alimentation des troupeaux, a été mené dans le périmètre irrigué du Gharb (au nord-ouest du pays). Les rations alimentaires effectivement ingérées par les vaches en lactation dans six élevages ont été reconstituées et pesées et leur contenu en nutriments (unités fourragères lait [UFL], protéines digestibles dans l'intestin lorsque l'azote est limitant [PDIN] et protéines digestibles dans l'intestin lorsque l'énergie est le facteur limitant [PDIE]) a été déterminé à partir des valeurs tabulées des aliments $(7,11)$. Ceci a été effectué à deux reprises en 2013 : au début du printemps, au moment d'une relative abondance alimentaire, et au milieu de l'été, en période de soudure. En parallèle, dans les six troupeaux, le potentiel moyen quotidien de production de lait par vache traite a été évalué à chacune de ces deux périodes. Cette valeur quotidienne nécessite au préalable de connaître le potentiel annuel de lactation, qui dépend des caractéristiques génétiques des troupeaux étudiés. Dans cette optique, un potentiel de production annuelle de $8000 \mathrm{~kg}$ a été retenu pour les troupeaux constitués exclusivement de vaches importées Holstein, $7000 \mathrm{~kg}$ pour les troupeaux avec des vaches Montbéliardes, et $5000 \mathrm{~kg}$ pour les vaches de type croisé. Dans un troupeau composite incluant des vaches croisées et des vaches de pure race Holstein, une valeur annuelle moyenne de 6000 à $6500 \mathrm{~kg}$ de lait a été retenue selon l'importance relative des différents types génétiques dans l'effectif total du troupeau. Le calcul du potentiel moyen de lactation quotidien nécessite de connaître, outre la constitution génétique des troupeaux, leur stade physiologique moyen. Ceci correspond au stade de lactation du troupeau déterminé au cours de la période de l'étude (de mars à juillet). Cet indicateur moyen mensuel pour chaque troupeau a été calculé par la formule suivante :

durée moyenne de lactation par vache et par mois $=\Sigma$ nombre de jours de traite pour la vache $k$ et le mois $j$ / nombre de vaches traites durant le mois $j$ x 30,6

$\mathrm{Au}$ final, pour les deux périodes d'évaluation des performances des vaches laitières (mars et juillet), une valeur moyenne du potentiel laitier quotidien a été déterminée à partir des modèles de lactation standard pour les races considérées dans cette étude (30). Cette valeur a ensuite été comparée à la production laitière réelle de la journée et le manque à gagner (différence entre les productions potentielle et réelle) a été précisé. En outre, l'analyse des contenus en nutriments des rations a permis d'interpréter les différences constatées entre le potentiel laitier et la production quotidienne réelle. Il s'agissait de déterminer si la contrainte alimentaire pouvait effectivement expliquer les performances obtenues, ce qui a permis de renforcer l'évaluation des systèmes fourragers et d'aborder les pistes d'amélioration.

\section{RESULTATS}

\section{Structuration du prix de revient du lait de vache}

L'analyse de la structure du prix de revient du lait a été variable selon la méthode de calcul considérée. En prenant en compte l'ensemble des charges, y compris amortissements et rémunération 
de la main-d'œuvre familiale (première méthode), le prix moyen de revient du kilogramme de lait a été de $0,37 €$. Il a varié de $0,30 € / \mathrm{kg}$ dans la région de la Moulouya (nord-est du Maroc) à $0,44 € / \mathrm{kg}$ dans le Tadla (centre du pays) (tableau III). Ces valeurs moyennes régionales se caractérisent par une grande variabilité lorsque l'écart type leur est supérieur. Quasiment toutes les moyennes ont été supérieures au prix de vente du lait au départ de la ferme, soulignant aussi que l'élevage bovin était déficitaire au moment de l'enquête.

Les dépenses de production ont été largement dominées par les charges alimentaires qui représentaient un peu plus de la moitié du total, suivies de la main-d'œuvre, des amortissements, des frais divers (litière, carburant et lubrifiants), enfin des charges liées aux soins vétérinaires et aux inséminations (figure 2). Les ventes de bovins (veaux et vaches de réforme) représentaient en moyenne un revenu de près de $0,09 € / \mathrm{kg}$ de lait et ont été déduites

\section{Tableau III}

Prix de revient (coût de production) du lait selon les deux méthodes de calcul utilisées et prix du lait au départ de la ferme $(€ / \mathrm{kg})$

$\begin{array}{lccc}\text { Région } & \begin{array}{c}\text { Moyenne du } \\ \text { prix de revient }\end{array} & \begin{array}{c}\text { Moyenne du } \\ \text { prix de revient } \\ \text { monétaire }{ }^{1}\end{array} & \begin{array}{c}\text { Moyenne du } \\ \text { prix du lait au } \\ \text { départ de la ferme }\end{array} \\ \text { Ben Slimane } & 0,38 \pm 0,64 & 0,31 \pm 0,52 & 0,24 \\ \text { Gharb } & 0,35 \pm 0,53 & 0,27 \pm 0,46 & 0,23 \\ \text { Loukkos } & 0,41 \pm 0,41 & 0,20 \pm 0,32 & 0,30 \\ \text { Moulouya } & 0,30 \pm 0,45 & 0,18 \pm 0,26 & 0,32 \\ \text { Rabat-Salé } & 0,34 \pm 1,26 & 0,17 \pm 0,48 & 0,30 \\ \text { Souss-Massa } & 0,43 \pm 0,44 & 0,24 \pm 0,26 & 0,35 \\ \text { Tadla } & 0,44 \pm 0,37 & 0,31 \pm 0,29 & 0,25 \\ \text { Moyenne } & 0,37 \pm 0,55 & 0,23 \pm 0,39 & 0,34 \\ \text { échantillon } & & & \end{array}$

${ }^{1}$ Amortissements et main-d'œuvre non compris

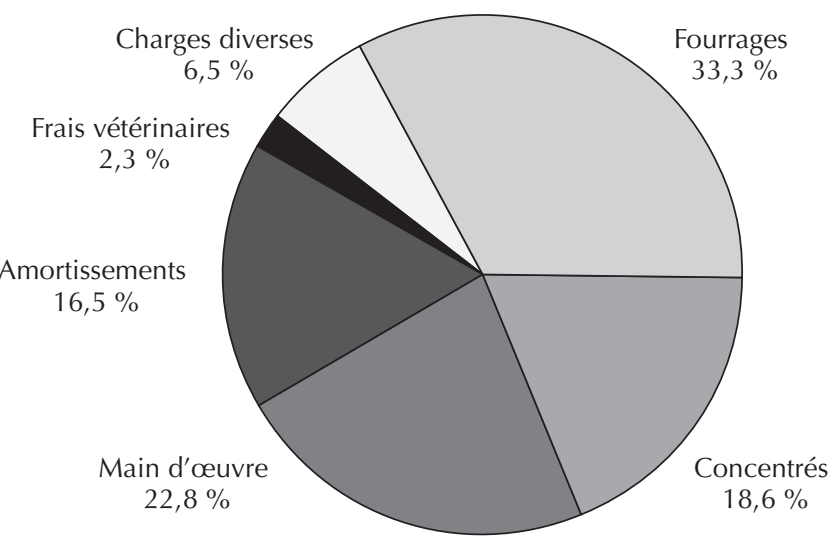

Figure 2 : contribution des différentes charges au prix de revient du lait selon la méthode de calcul qui intègre, outre les dépenses courantes (aliments, main d'œuvre extra-familiale salariée, frais vétérinaires et d'insémination, électricité, et énergie fossile), les amortissements des investissements et la rémunération de la main-d'œuvre familiale. du prix de revient du lait. Ces données moyennes cachent néanmoins de grandes disparités entre les fermes, avec de nombreux cas d'étables rentables. Sur les 86 exploitations étudiées, 28 étaient rentables, ce qui s'explique d'abord par un rendement laitier plus élevé que la moyenne (> $15 \mathrm{~kg} /$ vache/jour) comme le montre la figure 3. En outre, un usage raisonné des intrants, notamment à travers le rationnement, voire par un mode d'élevage utilisant très peu de concentrés et basé sur le pâturage après récolte sur des parcelles cultivées et sur le pâturage des herbes spontanées, permet d'abaisser le prix de revient du lait.

Le coût de production monétaire du lait, qui n'incluait pas la rémunération des amortissements et de la main-d'œuvre familiale (deuxième méthode), a été en moyenne de $0,23 € / \mathrm{kg}$. Ainsi, les charges non monétaires de production (amortissements et maind'œuvre familiale) équivalaient en moyenne à 38,5 p. 100 des charges totales de production. Les valeurs extrêmes des coûts de production monétaires ont été relevées dans les régions de RabatSalé $(0,17 € / \mathrm{kg}$ comme minimum) et du Tadla $(0,31 € / \mathrm{kg}$ comme maximum). Sous cet angle, la production de lait a semblé rentable pour 59 des 86 exploitations. Toutefois, ce prix de revient monétaire moyen est resté supérieur au prix du lait au départ de la ferme en vigueur dans trois régions : Ben Slimane, Gharb et Tadla (tableau III). Dans ces régions où le prix monétaire est resté supérieur au prix au départ de la ferme, les coûts alimentaires étaient élevés : soit les exploitations utilisaient de grandes quantités d'aliments concentrés (cas de la zone pluviale de Ben Slimane), soit elles avaient subi les effets d'inondations de printemps (cas du Gharb), soit le coût d'installation des fourrages était élevé (cas de la luzerne au Tadla). L'élevage bovin laitier est ainsi apparu fortement déficitaire dans les exploitations familiales relevant de ces trois régions.

\section{Evaluation des manques à gagner en élevage laitier conventionnel}

Les caractéristiques des six élevages concernés par le deuxième volet de l'étude sont détaillées dans le tableau IV. Ces exploitations, situées dans le périmètre irrigué du Gharb, représentaient l'essentiel des types de production bovine décrits dans cette région. Leurs stratégies fourragères s'appuyaient sur la culture du bersim (Trifolium alexandrinum) pour l'alimentation des vaches. Leurs rations étaient complétées par des pailles et chaumes de céréales (exploitations 2, 4, 5 et 6), et par des cultures d'été comme le maïs et la luzerne, afin de subvenir à leurs besoins à la fin du cycle du bersim (exploitations 1 et 3 ).

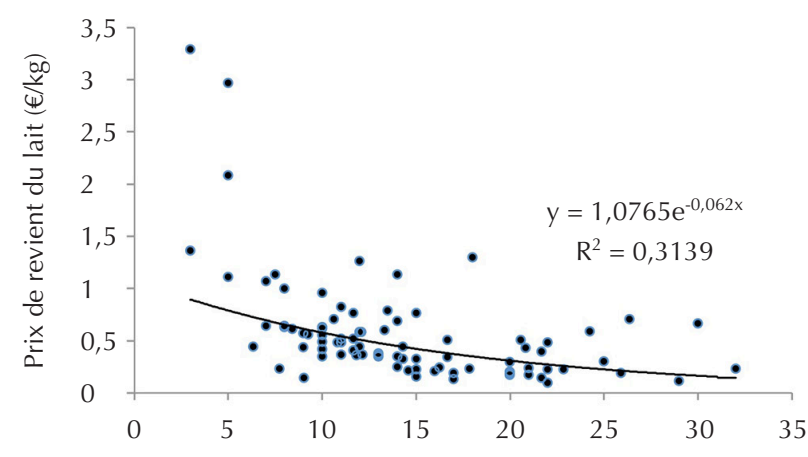

Figure 3 : relations entre le rendement laitier ( $\mathrm{kg} /$ vache/jour) et le prix de revient du lait. 
Les rations distribuées dans tous les troupeaux sans exception et au cours des deux saisons où l'étude a été réalisée ont été insuffisantes et/ou déséquilibrées, comme le montrent les deux exemples suivants : dans le premier, une ration de début de printemps basée sur de l'ensilage de maïs a été distribuée à un troupeau de vaches de type croisé et de race Holstein (exploitation 1), et dans le deuxième une ration estivale (à la fin du cycle du bersim) a été distribuée à un troupeau avec des vaches Holstein (exploitation 2). Les apports nutritionnels de ces deux rations sont reportés dans le tableau V. La principale caractéristique de ces deux rations était leur contenu insuffisant en matière sèche (MS), puisque pour des vaches capables d'ingérer près de $15 \mathrm{~kg}$ de MS par jour, les apports maximaux ne dépassent pas 9,6 kg.

Par ailleurs, dans les deux cas des déséquilibres nutritionnels manifestes ont été identifiés. Dans l'exploitation 1, l'ensilage de maïs était mal valorisé car complémenté exclusivement avec du son de riz, aliment dont la teneur en azote (PDIN) est limitée. Au final, les apports totaux de la ration en PDIN étaient inadaptés au regard du potentiel (20 kg de lait/vache/jour) et limitaient le rendement laitier moyen par vache traite à seulement $14 \mathrm{~kg}$ par jour. Dans l'exploitation 2 en été, la ration était composée de mauvais foin de bersim, de son de blé et d'aliment composé. A ce niveau aussi, une faible disponibilité de fourrages a été enregistrée : à peine $7,5 \mathrm{~kg}$ de MS par vache et par jour. Un déséquilibre a aussi été enregistré : les apports énergétiques limitaient le rendement laitier moyen par vache traite à $14 \mathrm{~kg}$ de lait par jour, alors que le potentiel était de $28 \mathrm{~kg}$, suite à deux nouveaux vêlages (dans un troupeau de trois vaches). Pire, un gaspillage azoté de $301 \mathrm{~g}$ de PDIN a été constaté ; il n'a pas été valorisé, en raison d'apports énergétiques limités, ce qui pouvait nuire à la santé des vaches et à l'environnement physique de l'exploitation, sans omettre les conséquences sur l'économie de la production.

Au final, les manques à gagner moyens estimés à partir des deux évaluations de rations distribuées aux vaches laitières dans les six élevages ont été déterminés (tableau VI). Le déficit moyen pour les six étables et lors des deux contrôles saisonniers a été de $5 \mathrm{~kg}$ de lait par vache et par jour, soit le tiers de la quantité réelle de lait produit. Les déficits les plus prononcés ont été observés dans les troupeaux avec le potentiel le plus élevé (au-delà de $20 \mathrm{~kg}$ de lait par vache et par jour en moyenne), ce qui signifie que les vaches à haut potentiel (surtout les Holstein en début de lactation) ont été moins bien valorisées dans les exploitations étudiées que les vaches à potentiel limité (de type croisé ou en fin de cycle de lactation).

Ces manques à gagner individuels ramenés à un nombre de 305 jours de lactation par an et à un prix du lait moyen au départ de la ferme de $0,23 € / \mathrm{kg}$ a permis de calculer l'équivalent de ce manque à gagner par exploitation en chiffres d'affaire. Ceux-ci ont varié d'une valeur minimale de $147 €$ par vache et par an dans l'exploitation 3 à $589 €$ dans l'exploitation 1 . Ces valeurs de manques à gagner n'incluaient pas les effets économiques des déséquilibres alimentaires sur la santé reproductive des vaches, qui n'ont pu être précisés dans cette étude.

\section{Tableau IV}

Caractéristiques structurelles des six élevages étudiés

\begin{tabular}{|c|c|c|c|c|c|c|}
\hline \multirow[t]{2}{*}{ Exploitation } & \multicolumn{3}{|c|}{ Surface fourragère (ha) } & \multirow[t]{2}{*}{ Céréales (ha) } & \multirow[t]{2}{*}{ Vaches traites } & \multirow[t]{2}{*}{ Potentiel laitier estimé (kg/an) } \\
\hline & Bersim & Maïs & Luzerne & & & \\
\hline 1 & 10,0 & 30,0 & - & 70,0 & 20 & 6000 \\
\hline 2 & 1,3 & - & - & 3,2 & 3 & 8000 \\
\hline 3 & 5,0 & 5,0 & 3,0 & 12,0 & 15 & 5000 \\
\hline 4 & 7,0 & - & - & 2,5 & 6 & 6000 \\
\hline 5 & 7,0 & - & - & 2,0 & 6 & 6500 \\
\hline 6 & 28,0 & - & - & 7,0 & 7 & 7000 \\
\hline
\end{tabular}

\section{Tableau V}

Evaluation des rations alimentaires des vaches laitières et comparaison aux besoins estimés pour la production réelle observée

\begin{tabular}{|c|c|c|c|c|c|c|}
\hline \multirow[t]{2}{*}{ Exploitation } & \multirow[t]{2}{*}{ Vaches traites } & \multirow{2}{*}{$\begin{array}{l}\text { Potentiel estimé } \\
\text { (kg/vache/jour) }\end{array}$} & \multirow{2}{*}{$\begin{array}{c}\text { Ration } \\
\text { (kg MS/vache/jour) }\end{array}$} & \multirow{2}{*}{$\begin{array}{l}\text { Rendement laitier réel } \\
\text { (kg/vache/jour) }\end{array}$} & \multicolumn{2}{|c|}{ Bilan ${ }^{1}$} \\
\hline & & & & & UFL & g PDI \\
\hline 1 (printemps) & 12 & 20,0 & $\begin{array}{l}\text { Ensilage de maïs }(9,6) \\
\quad \text { Son de riz }(4,6)\end{array}$ & 14 & $+1,47$ & -115 \\
\hline 2 (été) & 3 & 28,0 & $\begin{array}{l}\text { Foin de bersim }(7.5) \\
\text { Aliment composé }(4,0) \\
\text { Son de blé }(2,0)\end{array}$ & 14 & $+0,12$ & +301 \\
\hline
\end{tabular}

MS : matière sèche ; UFL : unités fourragères lait

${ }^{1}$ Différence entre les apports en nutriments (UFL et min. des valeurs PDIN et PDIE) de la ration et les besoins estimés pour la production réalisée (rendement laitier réel) PDIN : protéines digestibles dans l'intestin lorsque l'azote est limitant ; PDIE : protéines digestibles dans l'intestin lorsque l'énergie est le facteur limitant 


\section{Tableau VI}

Rendements laitiers quotidiens potentiels et réels, et manque à gagner ( $\mathrm{kg} / \mathrm{vache} / \mathrm{jour})$

\begin{tabular}{lcccc} 
Exploitation & $\begin{array}{c}\text { Vaches } \\
\text { traites }\end{array}$ & \multicolumn{2}{c}{$\begin{array}{c}\text { Rendement laitier } \\
\text { (moyenne } \pm \text { écart type) }\end{array}$} & $\begin{array}{c}\text { Manque } \\
\text { à gagner } \\
(\mathbf{E}-\mathbf{R})\end{array}$ \\
\cline { 3 - 4 } & & $\begin{array}{c}\text { Potentiel } \\
\text { estimé }(\mathbf{E})\end{array}$ & $\begin{array}{c}\text { Réel } \\
(\mathbf{R})\end{array}$ & \\
\hline 1 & 14 & $20,0 \pm 0,7$ & $11,6 \pm 3,8$ & 8,4 \\
2 & 2 & $22,8 \pm 5,8$ & $15,5 \pm 1,6$ & 7,3 \\
3 & 9 & $15,1 \pm 0,7$ & $13,0 \pm 2,1$ & 2,1 \\
4 & 3 & $22,5 \pm 3,4$ & $17,2 \pm 4,9$ & 5,3 \\
5 & 6 & $23,5 \pm 2,8$ & $19,8 \pm 5,5$ & 3,7 \\
6 & 6 & $18,5 \pm 1,9$ & $15,3 \pm 2,3$ & 3,2 \\
\hline Moyenne & 10 & $20,4 \pm 2,5$ & $15,4 \pm 3,4$ & 5,0
\end{tabular}

\section{DISCUSSION}

Les deux volets de cet article ont visé à porter un regard complémentaire sur les difficultés actuelles de l'élevage bovin au Maroc, exacerbées par les évolutions récentes du contexte général où il est pratiqué. Dans la première partie, le prix de revient du lait bovin a été évalué. Si l'on intègre la totalité des charges réellement assumées par les éleveurs (y compris les charges d'amortissement des investissements et la rémunération de la main-d'œuvre familiale), le prix moyen de revient du lait atteignait alors $0,37 €$ par kilogramme. Cette valeur est supérieure à ce qui est rapporté dans d'autres études en Afrique et notamment au Maroc $(17,26)$ et peut s'expliquer justement par la prise en compte des charges d'amortissement, notamment des vaches. En effet, le coût de l'élevage des primipares de remplacement constitue une composante importante du prix de revient du lait (8). Les résultats obtenus confirment aussi que le prix de revient du lait au Maroc est en moyenne l'un des plus élevés en Afrique (9) ; ceci s'explique par des charges alimentaires lourdes dues à la dominance de systèmes en « zéro pâturage ». Par ailleurs, le prix de revient du lait a été dans presque toutes les régions étudiées supérieur au prix de vente du lait au départ de la ferme. Ces chiffres impliquent une vulnérabilité économique marquée des élevages bovins laitiers au Maghreb de manière générale (18), et au Maroc plus particulièrement (26). Ces constats rejoignent aussi, mais dans un contexte relativement différent, des résultats récents obtenus en France et en Allemagne, qui dévoilent les difficultés économiques des élevages bovins laitiers spécialisés, allant jusqu'à réclamer un prix de vente du lait au départ de la ferme de $0,45 €$ par litre pour garantir la résilience de ce secteur d'activité $(5,6)$.

Les chiffres moyens cachent néanmoins une importante variabilité des résultats économiques entre fermes, avec 28 situations rentables sur 86. On constate que les coûts de production étaient les plus bas là où les rendements moyens en lait par vache étaient les plus élevés (au-delà de $15 \mathrm{~kg}$ de lait par jour), ce qui permet de « diluer les frais fixes d'élevage » (32). Les situations rentables correspondaient aussi à des cas d'élevages plus extensifs, qui utilisaient moins d'aliments concentrés achetés et où les vaches valorisaient des ressources alimentaires gratuites, comme l'herbe des jachères ou des parcours forestiers (cas des régions du nord du pays, comme le Gharb, le Loukkos ou Rabat-Salé). Toutefois, ces ressources alimentaires issues de pâturage de ressources non semées ont un coût social évident, dès lors qu'elles impliquent un volume de travail conséquent (23). Ces constats s'accordent avec des travaux montrant la possibilité d'influer sur la rentabilité de l'élevage bovin par une diminution des achats de concentrés (4) ou par une maîtrise poussée de leur conversion en lait (31).

En outre, la situation de déficit moyen des exploitations bovines laitières doit aussi être relativisée par rapport à la saison où cette étude a été réalisée, c'est-à-dire au début du printemps jusqu'à l'été, soit au moment le plus favorable de l'année pour la production de fourrages. Ceci implique que les prix de revient du lait calculés ici sont probablement inférieurs aux prix de revient du lait en fin de période estivale et automnale, lors de la soudure alimentaire. Des travaux complémentaires sont donc nécessaires pour caractériser l'évolution sur le long terme du prix de revient du lait au Maroc, en vue d'y intégrer les effets des variations climatiques saisonnières et interannuelles.

Le coût de production du lait qui ne tient pas compte des amortissements et de la main-d'œuvre familiale a été inférieur au prix de vente du lait au départ de la ferme dans quatre des sept régions, ce qui indique que l'élevage y était plus rentable que dans les autres régions.

Au final, les deux volets de ce travail soulignent la fragilité financière des exploitations laitières familiales. En effet, nombre de ces exploitations fonctionnent à perte et ne peuvent pas reconstituer de manière durable leur outil de production ni rémunérer au Smag le travail des membres de la famille. Or, il est avéré que les investissements en élevage laitier sont particulièrement lourds (machines, bâtiments, matériel, équipements d'irrigation, etc.) et fragiles (notamment les vaches, dont les carrières peuvent être écourtées suite à un accident), ce qui implique de disposer en permanence de fonds pour en assurer le renouvellement, générant ainsi des charges que seul le lait permet de récupérer (4). Quant au travail, il s'érige inexorablement en contrainte majeure dans l'élevage laitier, en raison des soins quotidiens exigés par les troupeaux (2). Ainsi, même si la souplesse d'usage de l'emploi de main-d'œuvre familiale octroie aux exploitations paysannes des opportunités remarquables de résilience (23), il n'est pas exclu que sa rémunération insuffisante conduise les exploitations à changer d'activité. Ceci pourrait compromettre le développement futur de la production laitière au Maroc.

Le deuxième volet de l'article présente une estimation des manques à gagner théoriques de six élevages bovins laitiers de la région irriguée du Gharb. La comparaison entre le potentiel moyen quotidien de production, estimé sur la base de la caractérisation du statut physiologique des vaches traites au cours de deux saisons distinctes (printemps et été), et la quantité réelle de lait produite, renseigne sur ces manques à gagner. Pour expliciter davantage les contreperformances identifiées, les relations entre la production effective et les contenus en nutriments (énergie et azote) des rations ingérées ont été précisées. Les résultats confirment les tendances lourdes identifiées dans des diagnostics antérieurs de l'état de la production laitière au Maroc, à savoir des rations alimentaires souvent insuffisantes (pas assez de MS) puisqu'une vache de poids vif de $600 \mathrm{~kg}$ peut ingérer jusqu'à $15 \mathrm{~kg}$ de MS de fourrages à haute digestibilité (3), alors que les valeurs enregistrées dans cette étude ne dépassent pas $10 \mathrm{~kg}$. Parmi les causes expliquant cette faible disponibilité de biomasse alimentaire, des charges animales élevées et des problèmes agronomiques dans la conduite des cultures fourragères peuvent être incriminés (19). En outre, l'étude a montré des problèmes récurrents de rations déséquilibrées, du fait de la méconnaissance des éleveurs des contenus en nutriments 
des rations et de leurs évolutions, ainsi que des changements des besoins des vaches selon leur stade moyen de lactation et leur potentiel génétique (28).

Les spécificités des systèmes fourragers dans la zone étudiée, avec comme pivot principal le bersim, induisent un excès d'azote lors de sa période de disponibilité maximale. Mais à la fin de son cycle de production, les teneurs en azote des rations chutent, ce qui déprécie rapidement le rendement laitier si une complémentation raisonnée n'est pas adoptée (18). De même, avec des rations basées sur des fourrages riches en énergie et pauvres en azote, comme l'ensilage de maïs, il est évident que si la complémentation en concentrés ne pallie pas ces manques, le rendement laitier est aussi limité.

Par ailleurs, l'étude montre que les décalages entre les rendements en lait potentiel et effectif sont les plus prononcés dans les exploitations qui possèdent les vaches aux potentialités génétiques les plus élevées. Ce constat conforte d'autres travaux qui remettent en cause les introductions massives de vaches à fortes capacités laitières si, en parallèle, une action de fond d'appui et de conseil n'est pas entreprise pour promouvoir leur alimentation équilibrée et suffisante (16). Les manques à gagner calculés dans les six différentes étables ont varié en moyenne de 2,1 à 8,7 kg de lait par vache traite et par jour, ce qui correspondait à une perte de chiffre d'affaires variant de 147 à $589 €$ par vache et par lactation standard de 305 jours. Pareils chiffres expliquent largement les difficultés économiques des élevages, comme le montrent les résultats présentés dans le premier volet de cet article. En outre, les manques à gagner identifiés dans cette étude ne concernent que les volumes de lait non produits. Or, les déséquilibres des rations alimentaires, notamment entre l'énergie et l'azote, sont aussi à l'origine de problèmes métaboliques des vaches qui peuvent induire des retards de la reproduction, voire l'infécondité, et déboucher sur des réformes précoces des vaches (13). Il est évident que ces déboires de la reproduction bovine ont aussi des conséquences marquées sur l'économie des exploitations bovines laitières (10).

\section{CONCLUSION}

La présente étude confirme les difficultés financières croissantes que doivent affronter les éleveurs de bovins laitiers au Maroc. Dans un contexte de volatilité croissante des prix des matières premières importées pour l'alimentation animale, les éleveurs revendiquent avant tout l'augmentation du prix de vente du lait ou prix au départ de la ferme. A titre d'exemple, dans un article intitulé "Souss Massa Draa » du quotidien marocain L'Economiste du 30 août 2013, les éleveurs réclament une hausse d'un dirham par litre. Le présent travail confirme la légitimé de ces revendications et montre qu'en incluant dans la méthode de calcul du prix de revient du lait toutes les charges y compris la main-d'œuvre familiale et les amortissements des investissements, cette production n'est pas rentable.

Le deuxième volet du travail complète la caractérisation de l'économie de la production reflétée par le prix de revient du lait, en ce sens qu'il souligne les manques à gagner issus de pratiques d'alimentation des troupeaux inadaptées. Les suivis des apports nutritionnels des rations ingérées et leur mise en regard du potentiel de production porte un éclairage édifiant sur l'origine des manques à gagner : des rations insuffisantes (pas assez de MS dans les fourrages) et de surcroît souvent déséquilibrées (carence relative en azote ou en énergie). Au final, les conclusions issues de ces deux travaux montrent que les évolutions récentes du contexte général de l'élevage bovin exacerbent les difficultés économiques de la production. Pour y pallier, des changements dans les orientations actuelles sont nécessaires, pour le maintien de cette activité et pour la sécurisation des approvisionnements des laiteries.
A cet égard, une augmentation de près de 10 p. 100 du prix du lait de boisson à la consommation a été enregistrée en été 2013 (de $0,57$ à $0,62 € / L)$ suivie d'une autre de même ampleur au printemps 2014 sur les dérivés laitiers les plus courants (yaourts et préparations fromagères), avec comme argument principal, la répercussion d'une part importante de ces hausses vers l'amélioration du prix du lait au départ de la ferme qui avait stagné durant les quinze dernières années. Apparemment, les pouvoirs publics et les industriels les plus influents n'ont pris cette décision d'augmenter la rémunération du kilogramme de lait au départ des exploitations d'élevage qu'après avoir effectivement ressenti les tensions autour de la question cruciale de la répartition de la valeur dans la chaîne. Pareil signal reflète aussi sûrement la volonté de sécuriser les approvisionnements en évitant que de nombreux éleveurs, excédés de subir des pertes économiques répétées, ne renoncent à la production bovine, ce qui précipiterait la chute de la livraison du lait.

Outre la question des prix du lait le long de la chaîne, un autre volet fondamental d'intervention pour promouvoir la durabilité économique des exploitations bovines est lié à l'appui technique nécessaire pour assurer l'augmentation de la productivité. Pour ce faire, un accompagnement des élevages qui le désirent vers une maîtrise accrue des techniques de base (rationnement, connaissance des apports des aliments, aussi bien les fourrages que les concentrés, et de l'évolution des besoins des vaches selon leur stade moyen de lactation) est nécessaire. A ce propos, les pouvoirs publics en charge du secteur agricole envisagent de créer une nouvelle organisation du conseil agricole qui travaillerait en partenariat avec des opérateurs privés. Cette instance devra aussi intégrer dans ses prérogatives des interventions de terrain permettant de contribuer à améliorer le sort des exploitations laitières familiales de taille réduite qui demeurent les acteurs incontournables de la fourniture de lait et de viande bovine au Maroc.

Enfin, la présente étude n'ayant pas pu prendre en compte les fermes laitières de plus de 30 vaches ainsi que les très grandes exploitations spécialisées qui s'installent dans le cadre de la nouvelle orientation agricole adoptée par le Maroc en 2009 ( «Plan Maroc Vert »), d'autres travaux seront nécessaires pour comparer les performances de ces grandes exploitations avec celles des petites fermes familiales.

\section{Remerciements}

Ces travaux ont été effectués dans le cadre du projet Crop/Livestock Integration in The MEDiterranean (CLIMED). Les auteurs souhaitent remercier les cadres techniques de l'Association nationale des éleveurs de bovins (ANEB) au Maroc pour leur soutien logistique à la réalisation de ces deux études de terrain. Merci en particulier aux éleveurs rencontrés durant ces travaux pour leur accueil chaleureux et leurs réponses aux nombreuses sollicitations imposées par les protocoles de recherche.

\section{BIBLIOGRAPHIE}

1. ADA, 2011. La situation de I'agriculture au Maroc. Rabat, Maroc, Agence du développement agricole, 202 p. www.agriculture.gov.ma/ sites/default/files/SAM9-2011.pdf

2. AUBRON C., COCHET H., BRUNSCHWIG G., MOULIN C.-H., 2009. Labor and its productivity in Andean dairy farming systems: a comparative approach. Hum. Ecol., 37: 407-419.

3. CASTILLO A.R., TAVERNA A.M., PAEZ R.R., CUATRIN A. COLOMBATTO D., BARGO F., GARCIA M.S., GARCIA P.T., CHAVEZ M., BEAULIEU A.D., DRACKLEY J.K., 2006. Fatty acid composition of milk from dairy cows fed fresh alfalfa based diets. Anim. Feed Sci. Technol., 131: $241-254$.

4. CORDONNIER P., 1986. Economie de la production laitière. Paris, France, Lavoisier, 218 p. 
5. EUROPEAN MILK BOARD, 2014. Combien coûte la production de lait ? Les coûts de la production de lait en France, édn 1 de 2002 à 2013 47 p. www.europeanmilkboard.org/fileadmin/Dokumente/Press_Release/ EMB-allgemein/2014/Etude_sur_le_cou\%CC\%82t_de_production_ laitie\%CC\%80re_en_France_FINAL_FR.pdf

6. EUROPEAN MILK BOARD, 2014. Combien coûte la production de lait ? Calcul des coûts de la production laitière en Allemagne de 2002 à 2012, 35 p. www.europeanmilkboard.org/fileadmin/Dokumente/Press_ Release/EMB-allgemein/2013/etude_couts_de_production_FR.pd

7. GUESSOUS F., 1991. Production fourragère et systèmes animaux. Rabat, Maroc, Institut agronomique et vétérinaire Hassan II / Actes Editions, $128 p$

8. HEINRICHS A.J., JONES C.M., GRAY S.M., HEINRICHS P.A., CORNELISSE S.A., GOODLING R.C., 2013. Identifying efficient dairy heifer producers using production costs and data envelopment analysis. J. Dairy Sci., 96: 7355-7362.

9. HEMME T. Ed., 2012. Dairy report. Kiel, Germany, International Farm Comparison Network Dairy Research Center, 208 p.

10. INCHAISRI C., JORRITSMA R., VOS P.L.A.M., VAN DER WEIJDEN G.C., HOGEVEEN H., 2010. Economic consequences of reproductive performance in dairy cattle. Theriogenology, 74: 835-846.

11. JARRIGE R. Ed., 1988. Table des valeurs nutritionnelles des aliments In : Alimentation des bovins, ovins et caprins. Paris, France, INRA p. 351-443.

12. KIDD A.D., LAMERS J.P.A., FICARELLI P.P., HOFFMAN V., 2000. Privatising agricultural extension: caveat emptor. J. Rural Stud., 16: 95 102.

13. LEAN I.J., CELI P., RAADSMA H., MCNAMARA J., RABIEE A.R. 2012. Effects of dietary crude protein on fertility: Meta-analysis and meta-regression. Anim. Feed Sci. Technol., 171: 31-42.

14. MAPM, 2008. Plan Maroc Vert : premières perspectives sur la stratégie agricole. Rabat, Maroc, ministère de I'Agriculture et de la Pêche maritime. Www.vulgarisation.net/planmarocvert.pdf

15. MARA, 1975. Le plan laitier. Rabat, Maroc, ministère de l'Agriculture et de la réforme agraire, 115 p. + annexes

16. MORAN J.B., 2013. Addressing the key constraints to increasing milk production from small holder dairy farms in tropical Asia. Int. J. Agric. б Biosci., 2: 90-98.

17. NDAMBI O.A., HEMME T., 2009. An economic comparison of typical dairy farming systems in South Africa, Morocco, Uganda and Cameroon. Trop. Anim. Health Prod., 41: 979-994.

18. OLMOS COLMENERO I., BRODERICK G. A 2006. Effect of dietary crude protein concentration on milk production and nitrogen utilization in lactating dairy cows. J. Dairy Sci., 89: 1704-1712.
19. PAPY F., 1979. Eléments de réflexion sur la recherche fourragère au Maroc. Fourrages, 79 : 89-110.

20. REJEB GHARBI $F$, LAHSOUMI R GOUHIS R RACHED $Z$ 2007. Rentabilité économique de l'élevage laitier en Tunisie : cas des gouvernorats de I'Ariana et de Mahdia. Biotechnol. Agron. Soc. Environ., $11: 211-223$.

21. SAUVANT D., GIGER-REVERDIN S., MESCHY F., 2006. Le contrôle de l'acidose rumimale latente. Prod. Anim., $19: 69-78$.

22. SCHILLING J., KORBINIAN P.F., HERTIG E., SCHEFFRAN J., 2012. Climate change, vulnerability and adaptation in North Africa, with focus on Morocco. Agric. Ecosyst. Environ., 156: 12-26.

23. SRAIRI M.T., BAHRI S., KUPER M., 2013. Le travail et sa contribution aux stratégies d'adaptation de petites exploitations agricoles familiales mixtes d'élevage bovin/polyculture au Maroc. Biotechnol. Agron. Soc. Environ., $17: 463-474$

24. SRAIRI M.T., BENYOUCEF M.T., KRAIEM K., 2013. The dairy chains in North Africa (Algeria, Morocco and Tunisia): from self-sufficiency options to food dependency? Springer Plus, 2: 162. www.springerplus. com/content/2/1/162

25. SRAIRI M.T., CHOHIN-KUPER A., 2007. Consequences of market liberalization for the operators of the dairy subsector in Morocco. Rev. Elev. Méd. Vét. Pays Trop., 60: 177-187 [in French with English summary]

26. SRAIRI M.T., EL KHATTABI M., 2001. Evaluation économique et technique de la production laitière intensive en zone semi-aride au Maroc. Cah. Agric., 10 : 51-56.

27. SRAIRI M.T., ER-ROUSSE E.H., 2010. Cas d'un élevage laitier en zone pluviale au Maroc: des cultures au cheptel bovin, quelles voies d'amélioration? Fourrages, 201: 61-65.

28. SRAIRI M.T., KIADE N., LYOUBI R., MESSAD S., FAYE B., 2009. A comparison of dairy cattle systems in an irrigated perimeter and in a suburban region: case study from Morocco. Trop. Anim. Health Prod., 41: 835-843.

29. SRAIRI M.T., MOUSILI N., 2014. Effets des facteurs alimentaires et de la race bovine sur les performances de deux élevages laitiers en zone semi-aride au Maroc. Nature Technol., 10 : 50-55.

30. WILMINK, J.B.M., 1987. Adjustment of test-day milk, fat and protein yield for age, season and stage of lactation. Livest. Prod. Sci., 16: 335348.

31. WOLF C.A., 2010. Understanding the milk to feed price ratio as a proxy for dairy farm profitability. J. Dairy Sci., 93: 4942-4948.

32. WOLTER R., 1997. Alimentation de la vache laitière. Paris, France, France Agricole, 263 p.

Accepted 19 June 2014; Online publication 30 September 2015 


\section{Summary}

Sraïri M.T., Chergui S., Igueld H., Sannito Y. Performances of family dairy farms in Morocco: Arguments for improving farm milk price and technical support

Dairy cattle production in Morocco has been faced with a turbulent evolution over these past years. This activity yet contributes much to supply the dairy industry and urban markets as well as to maintain livelihoods in rural areas. The present study aimed to characterize the technical and economic performances of cattle farms in relation with constraints to overcome. In the first part, the milk cost price was analyzed through a survey conducted in 86 farms located in the main animal production areas of the country. Two calculus methods served to assess the profitability of this activity. In the second part, shortfalls caused by limited milk yields were characterized and linked to the rations fed to the lactating cows in six farms located in a favorable area (Gharb irrigation scheme) during two successive seasons (spring and summer). Results highlighted the limited profitability of the dairy farms and the scope for improvement of rationing, especially concerning cows with high genetic potential. Major requirements for sustainable dairy cattle farming in Morocco include i) advice to farmers to improve feeding practices, and ii) revaluation of the milk sales price at farm level.

Keywords: Cattle - Dairy cow - Milk yield - Production cost Ration - Sustainability - Profitability - Morocco.

\section{Resumen}

Sraïri M.T., Chergui S., Igueld H., Sannito Y. Rendimientos de los establecimientos lecheros familiares en Marruecos: argumentos para la mejoría del precio de la leche en la finca y del apoyo técnico

La cría bovina lechera en Marruecos a conocido estos últimos años una evolución agitada. Sin embargo, esta actividad contribuye fuertemente al abastecimiento de lecherías y de mercados urbanos, así como al mantenimiento de la vida en zona rural. El presente estudio tuvo como objetivo el de caracterizar los rendimientos técnicos y económicos de la cría bovina con respecto a los obstáculos que ha debido superar. En una primera parte, el precio de retorno (costo de producción) de la leche, fue estudiado sobre la base de una encuesta llevada a cabo en 86 establos distribuidos en las principales cuencas de cría de Marruecos. Dos métodos de cálculo permitieron la asesoría sobre la rentabilidad de esta actividad. En una segunda parte, se caracterizaron las pérdidas en ganancias derivadas de los rendimientos lecheros limitados y se relacionaron con las raciones ingeridas por las vacas en lactación en seis establos, durante dos estaciones sucesivas (primavera y verano), en una región propicia del país (perímetro irrigado del Gharb). Este trabajo subrayó la baja rentabilidad de las fincas bovinas y las márgenes de mejoramiento en materia de racionamiento, sobre todo para las vacas con alto potencial. La asesoría de los criadores que lleve al mejoramiento de las prácticas de alimentación de los animales y la revalorización del precio de venta de la leche en la finca constituyen dos condiciones importantes del mantenimiento durable de la cría bovina lechera en Marruecos.

Palabras clave: Ganado bovino - Vaca lechera - Rendimiento lechero - Costo de producción - Ración - Sostenibilidad Rentabilidad - Marruecos. 
